\title{
Coupling histone homeostasis to centromere integrity via the ubiquitin-proteasome system
}

\author{
Yuko Takayama*1 and Takashi Toda*2
}

\begin{abstract}
In many eukaryotes, histone gene expression is regulated in a cell cycle-dependent manner, with a spike pattern at $\mathrm{S}$ phase. In fission yeast the GATA-type transcription factor Ams2 is required for transcriptional activation of all the core histone genes during $S$ phase and Ams 2 protein levels per se show concomitant periodic patterns. We have recently unveiled the molecular mechanisms underlying Ams2 fluctuation during the cell cycle. We have found that Ams2 stability varies during the cell cycle, and that the ubiquitin-proteasome pathway is responsible for Ams 2 instability. Intriguingly, Ams2 proteolysis requires Hsk1-a Cdc7 homologue in fission yeast generally called Dbf4-dependent protein kinase (DDK)-and the SCF ubiquitin ligase containing the substrate receptor Pof3 F-box protein. Here, we discuss why histone synthesis has to occur only during $S$ phase. Our results indicate that excess synthesis of core histones outside $S$ phase results in deleterious effects on cell survival. In particular, functions of the centromere, in which the centromere-specific H3 variant CENP-A usually form centromeric nucleosomes, are greatly compromised. This defect is, at least in part, ascribable to abnormal incorporation of canonical histone $\mathrm{H} 3$ into these nucleosomes. Finally, we address the significance and potential implications of our work from an evolutionary point of view.
\end{abstract}

\section{Introduction}

The timely and selective proteolysis of proteins is essential for cell cycle control. Particularly, ubiquitin-proteasome pathway plays a pivotal role in cell cycle transition and progression [1]. Substrate proteins are ubiquitylated by the enzymatic cascade consisting of ubiquitin-activating enzyme (E1), ubiquitin-conjugating enzyme (E2), and ubiquitin ligase (E3) [2]. The E3 ligase determines the substrate specificity of the pathway. These ubiquitin transferase reactions result in the formation of polyubiquitin chains on substrates, which are recognised by the proteasome, followed by rapid irreversible degradation.

Nucleosomes comprise the repeated units of chromosomal DNAs wrapped around histone octarmers that consist of two sets of each of $\mathrm{H} 2 \mathrm{~A} / \mathrm{H} 2 \mathrm{~B}$ and $\mathrm{H} 3 / \mathrm{H} 4$ dimer. As DNA replication proceeds, new nucleosomes are formed. Thus, the timing of histone synthesis and DNA replication is coupled, by which newly synthesised histones are rapidly deposited onto replicated DNA [3].

\footnotetext{
*Correspondence: yutakaya@lsi.kurume-u.ac.jp, toda@cancer.org.uk

${ }^{1}$ Division of Cell Biology, Institute of Life Science, Kurume University, 1-1 Hyakunen-kohen, Kurume, Fukuoka 839-0864, Japan

2 Laboratory of Cell Regulation, London Research Institute, Cancer Research UK, 44 Lincoln's Inn Fields, London WC2A 3PX, UK

Full list of author information is available at the end of the article
}

In yeast, previous reports showed that increased histone levels lead to chromosome instability [4] and enhanced DNA-damage sensitivity [5]. Chromosomal instability has been recognised as a hallmark of human cancer $[6,7]$. However, how the cellular amount of histones is regulated is largely unknown at the molecular levels.

Proper chromosome segregation requires a physical connection between spindle microtubules and centromeric DNAs and this attachment occurs via the kinetochore. CENP-A is a centromere-specific histone H3 variant that is essential for kinetochore formation. CENP-A represents the most likely candidate for the epigenetic mark responsible for maintenance of centromere identity $[8,9]$. Several recent studies have identified proteins specifically involved in CENP-A loading and centromeric nucleosome formation [10-16]. Interestingly, the cell cycle regulated GATA-type transcription factor, Ams2 in Schizosaccharomyces pombe that is required for activation of S-phase specific core histone transcription [17], also promotes the centromeric localisation of CENP-A [18,19]. Ams2 protein levels accumulate at G1-S phase, which is regulated by the ubiquitin-proteasome pathway $[20,21]$. In this commentary we describe our recent work on the molecular mechanisms of how Ams2 levels are regulated throughout the cell cycle and the del- 
eterious consequences when this intricate system goes wrong.

\section{Discussion}

Ams2 ensures a cell cycle-specific transcriptional spike of core histone gene expression

Ams2 was originally identified as one of the multicopy suppressors of the temperature sensitive (ts) cnp1-1 mutant [19], defective in the centromere-specific histone H3 variant CENP-A [22]. Interestingly genomic sequences encompassing canonical histone $\mathrm{H} 4$ genes were also isolated as other multicopy suppressors from the same screening. This raised the possibility that Ams2 could be involved in transcriptional control of histone genes. Indeed Ams2 is a member of the GATA factors containing Daxx and zinc finger DNA binding motifs (Figure 1A).

Subsequent analysis explicitly showed that Ams2 is a major, if not the sole, transcription factor required for the S-phase specific transcriptional spike of all of the core histone genes [17]. Ams2 directly binds a consensus "AACCCT-box" that exists in the $5^{\prime}$ franking regions of these histone genes. Note that $\operatorname{cnp} 1^{+}$is not regulated by Ams2 nor does it contain the "AACCCT-box" in its promoter region. A genome-wide search for the "AACCCT" motif followed by binding experiments suggested that core histone genes would be the major, if not the sole, targets of Ams2 (note that only one gene, SPAC631.02 encoding a conserved bromodomain protein, contains the "AACCCT-box" in its 5' flanking region, to which Ams2 binds [17]).

A

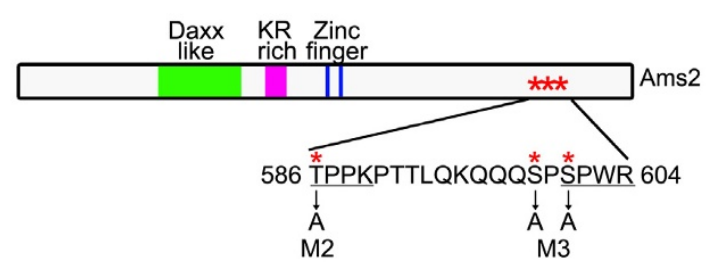

B

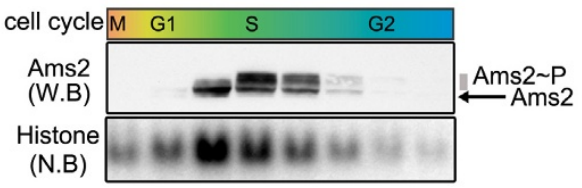

Figure 1 Ams 2 is a cell cycle-regulated GATA type transcription factor. (A) Schematic structure of Ams2. Structural domains homologous to Daxx (green), amino acid stretches rich in arginine and lysine (magenta) and zinc finger motif (blue) are depicted. Amino acid residues surrounding the phosphorylation sites (asterisks) (which are mutated to alanine in $\mathrm{M} 2$ and $\mathrm{M} 3$ mutants) and CDK phosphorylation consensus motifs (underlined) are also shown. (B) Fluctuation of Ams2 protein levels during the cell cycle. Wild-type cells were synchronised by centrifugal elutriation. The protein or RNA samples collected every 15 min were assayed by western (anti-Ams2, Ams2 W.B) or northern blotting (histone H4, Histone N.B).
In the absence of Ams2, transcriptional activation of histone genes does not occur during $\mathrm{S}$ phase, leading to very low levels of histone transcription throughout the cell cycle [17]. Cells with deletion of ams2+ manage to divide, but exhibit a number of defective phenotypes, including slower growth and frequent chromosome missegregation [19].

\section{Ams2 protein shows cell cycle-dependent oscillation due to varied protein stability}

In parallel with a spike of histone gene expression, Ams2 protein accumulates in the nucleus at $S$ phase $[17,19]$. In sharp contrast, little, if any of this protein is detected during mid G2 and M phase (see Figure 1B). That is, Ams2 protein appears to disappear from cells upon completion of S phase or at the beginning of G2 phase. ams $2^{+}$transcription is upregulated from G1 to $S$ phase, which is consistent with Ams2 protein accumulation during this period. However, this does not account for the rapid disappearance of Ams2 protein after S phase. Instead, this suggests the sudden destabilisation of Ams2 in G2 phase. Indeed the Ams 2 protein is unstable and rapidly degraded at G2 and M phase (half-life $\sim 20 \mathrm{~min}$ ), but is markedly stabilised in S phase (half-life $>60 \mathrm{~min}$ ). In G1 cells, the Ams2 is degraded, although at a much slower rate (half-life $\sim 40 \mathrm{~min}$ ). In summary, the Ams2 protein is unstable during $\mathrm{G} 2$ and $\mathrm{M}$ phase, partially stabilised in G1, and stable in S phase. Changes in protein stability coupled with transcriptional regulation, therefore, account for oscillations of Ams2 levels during the cell cycle.

\section{Ams2 protein is degraded via the SCFPof3-proteasome pathway}

Regulation of protein stability through the ubiquitin-proteasome pathway is a key mechanism underlying various cellular processes [1]. To address the involvement of this pathway in Ams2 stability, we examined the half-life of this protein in proteasome mutants. As suspected, Ams2 degradation during G2 phase was substantially suppressed, which was accompanied by massive accumulation of ubiquitylated Ams2.

There are two ubiquitin ligases that regulate cell cycle progression, Anaphase Promoting Complex/Cyclosome (APC/C) and Skp1-Cdc53/Cullin-1-F-box (SCF) [23,24]. As described earlier, Ams 2 protein is rapidly degraded in G2 phase. We posited that $\mathrm{APC} / \mathrm{C}$ is unlikely to be involved, as this ubiquitin ligase should be inactive at G2 phase, when Ams2 is degraded. This raised the possibility that the SCF is responsible. Subsequent analysis showed that this is in fact the case.

SCF consists of various subcomplexes, each of which contains different F-box proteins, components responsible for binding specific substrates. Our previous work 
and that from other laboratories showed that fission yeast contains 18 F-box proteins [25-27] (see Table 1). Three of these F-box proteins, Pop1, Pop2 and Pof3, play crucial roles in cell cycle progression and coordination [28-34]. Half-life analysis using individual mutants showed that the Ams2 protein was stabilised in the pof3 deletion mutant, but not in pop1 or pop 2 mutants. Furthermore, Pof3 did bound to Ams2, substantiating the suggestion that $\mathrm{SCF}^{\mathrm{Pof3}}$ is an E3 ligase responsible for degradation of Ams2.

\section{Cdc7 homologue Hsk1 is a kinase responsible for Ams2 degradation}

F-box proteins normally recognise phosphorylated substrates [35]. Indeed, Ams2 protein shows a cell cycle-specific band-shift on gel electrophoresis, in which a faster migrating form appears coincident with a peak of histone gene expression (Figure 1B). This faster form is then replaced by slower phosphorylated forms, followed by abrupt disappearance [17]. Half-life measurements of Ams2 using various $c d c$ mutants showed that $\mathrm{Cdk} 1 / \mathrm{Cdc} 2$ is not required for Ams2 instability (see below for details). As Ams2 phosphorylation occurs in S-phase, we examined the involvement of the S-phase kinase DDK
(Dbf4-dependent protein kinase, Cdc7 in budding yeast and Hsk1 in fission yeast). In the hsk1 mutant, Ams2 is hypo-phosphorylated and highly stabilised. This result suggested that Hsk1-DDK is a kinase responsible for Sphase phosphorylation of Ams2, which leads to $\mathrm{SCF}^{\text {Pof3 }}$ mediated proteolysis (see Figure 2 for scheme).

\section{Potential cooperative roles between CDK and DDK in Ams2 proteolysis}

In vitro kinase reaction showed that Ams2 is a substrate of Hsk1. Several subsequent experimentations narrowed down potential phosphorylation sites to three residues (586T, 599S and 601S, Figure 1A, asterisks). Mutagenesis analysis of these sites (M2, M3 and M2\&M3 in Figure 1A) indicated that $599 \mathrm{~S}$ and $601 \mathrm{~S}$ are phosphorylated by Hsk1-Dfp1 in vitro. Expression of individual mutants in fission yeast, followed by half-life analysis, supported that these threonine and serines are required for Ams2 phosphorylation and subsequent proteolysis.

It is notable that when a phospho mutant, i.e., a stable form of Ams2 (M2\&M3), is expressed from its native promoter, its mRNA levels are slightly but reproducibly decreased compared to those of wild-type Ams2. This result indicates that some monitoring system, possibly a

Table 1: Fission yeast F-box proteins

\begin{tabular}{|c|c|c|c|c|c|c|}
\hline \multirow[b]{2}{*}{ F-box protein } & \multirow[b]{2}{*}{ motifs } & \multirow[b]{2}{*}{ recognition protein } & \multicolumn{2}{|c|}{ homologue } & \multicolumn{2}{|l|}{ SCFI } \\
\hline & & & yeast & human & ${ }^{*}$ non-SCF & References \\
\hline Pop1 & WD40 & Cig2, Cdc18, Rum1 & Cdc4 & Fbw7 & SCF & {$[30,34,46]$} \\
\hline Pop2 & WD40 & Cig2, Cdc18, Rum1 & Cdc4 & Fbw7 & SCF & {$[30,34,46]$} \\
\hline Pof1 & WD40 & Zip1 & Met30 & $\beta \operatorname{TrCP}$ & SCF & [47] \\
\hline Pof2 & LRR & & Grr1 & $?$ & SCF & {$[25]$} \\
\hline Pof3 & LRR, TPR & Mcl1, Ams2 & Dia2 & $?$ & SCF & {$[25,28,48]$} \\
\hline Pof4 & none & & Ela1 & ElonginA & SCF & {$[25]$} \\
\hline Pof5 & none & & YDR360c & $?$ & SCF & {$[25]$} \\
\hline Pof6 & CAAX & & Rcy1 & $?$ & non-SCF & {$[49,50]$} \\
\hline Pof7 & none & & Hrt3 & $?$ & SCF & {$[25]$} \\
\hline Pof8 & none & & Ufo 1 & $?$ & non-SCF & {$[25,51]$} \\
\hline Pof9 & none & & YBR280c & $?$ & SCF & [51] \\
\hline Pof10 & WD40 & & YML088w & $?$ & SCF & {$[52]$} \\
\hline Pof11 & WD40 & & $?$ & $\beta \operatorname{TrCP}$ & SCF & [51]? \\
\hline Pof12 & none & & $?$ & $?$ & non-SCF & {$[25,51]$} \\
\hline Pof13 & none & & $?$ & $?$ & non-SCF & {$[25,51]$} \\
\hline Pof14 & none & Erg9 & $?$ & $?$ & SCF & {$[26]$} \\
\hline Fbh1 & DNA helicase & Atf1 & $?$ & Fbh1 & SCF & [53-58] \\
\hline SPAPB1A10.14 & none & & $?$ & $?$ & SCF & {$[51]$} \\
\hline
\end{tabular}

*Assignment of some F-box proteins (Pof6, Pof8, Pof12 and Pof13) as 'non-SCF' is based upon the fact that these F-box proteins, albeit binding Skp1, do not have the conserved proline residue within the F-box motif that is critical for direct interaction between F-box and Cullin-1 [59]. In fact, binding experiments showed this is the case [51]. The only exception is Pof9, which does not contain the proline residue, but it acts as a component of SCF. 


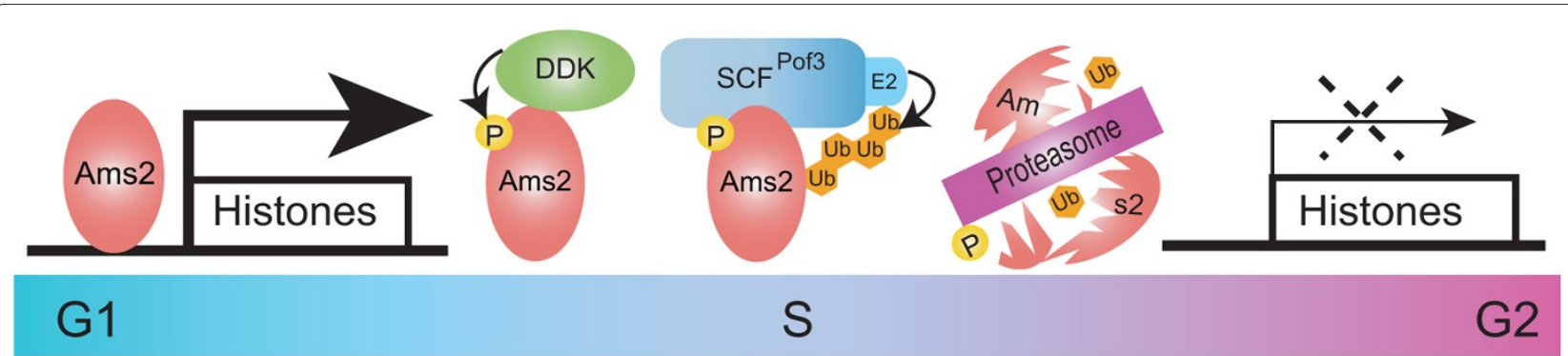

Figure 2 A model for the mechanism of Ams 2 protein oscillation. Ams 2 binds 5 ' flanking regions of core histone genes, thereby activating histone gene expression. Thereafter, Ams 2 is phosphorylated by DDK and its phosphorylated form is recognised by SCFPof3-ubiquitin ligase. Ubiquitylated Ams 2 is then degraded by the proteasome, resulting in repression of histone gene transcription.

feedback mechanism, would operate in maintaining the proper cellular levels of histone and/or Ams2. Further studies are necessary to uncover the identity of this regulatory loop.

Intriguingly, two sites of the above three residues are within the CDK consensus (586 $\underline{\text { PP }} \underline{589}$ and 601Pㅁ604, Figure 1A, underlined). As described earlier, our stability data, however, suggested that $\mathrm{Cdc} 2 /$ Cdk1 is not required for mobility shift of Ams2 phosphorylation or degradation. How could this apparent discrepancy be explained and what is the relationship between CDK consensus sites and DDK? Here, we posit two scenarios. One is that Hsk1 is capable of phosphorylating CDK consensus sites that are found in Ams2. As consensus phosphorylation sites for DDK remain to be determined, this notion cannot be ruled out. Another possibility, which is more appealing, is that CDK and DDK act in concert for Ams2 phosphorylation. According to this scenario, CDK would phosphorylate T586 and S601, thereby promoting subsequent S599 phosphorylation by Hsk1. Despite this, Cdc2-dependent phosphorylation may not be absolutely essential as far as activation of Hsk1 and phosphorylation of S599 and/or S601. It is tempting to speculate that CDK may play a role in Ams2 phosphorylation as a priming kinase for DDK, although this needs further scrutiny. It is also possible that in addition to ubiquitylation and degradation, individual phosphorylation by CDK and DDK could make distinct contributions toward Ams2 function, such as nuclear localisation and chromatin binding (see below). Further biochemical analysis will be necessary to draw solid conclusions regarding this issue.

\section{Spatiotemporal control of Ams2 stability during S phase}

Ams2 proteolysis requires phosphorylation by DDK, which is active during $S$ phase. However, as shown in Figure 3A, Ams2, both soluble and insoluble (chromatinbound), is stable when cells are arrested at $S$ phase with HU treatment. How can we reconcile this result? Interestingly, once released from HU block, Ams2, which is bound on chromatin, is stable, whereas phospho-Ams2 in the soluble fraction is selectively degraded (Figure 3A and 3B). Perhaps during early S phase or when DNA structure checkpoint is activated (triggered by HU treatment), Pof3 may be prevented from accessing phospho-Ams2. As $\mathrm{S}$ phase proceeds further, only soluble Ams2 is capable of interacting with Pof3, but chromatin-bound Ams2 is still not. We postulated that Ams2 stability is regulated in a dual manner, temporally via S-phase progression and spatially via its DNA/chromatin binding activity, which may be dependent on accessibility of SCFPof3 to Ams2.

\section{Centromeric nucleosomes are compromised by constitutive histone synthesis}

As described earlier, histone synthesis is under strict cell cycle control through Ams2. It would be thus of particular interest to see the physiological effects derived from constitutive Ams2 expression. Intriguingly, several expected and unexpected phenotypic consequences have been observed. It may not be surprising that excess Ams2 is harmful for cell growth with induced chromosome loss. This detrimental effect is exaggerated in the presence of the hsk1 mutation, consistent with the fact that this mutant already has increased levels of histones ascribable to Ams 2 stabilisation.

It is unexpected that chromatin architectures at the core centromere are dramatically altered. Micrococcal nuclease (MNase) experiments showed that the core centromere region, which normally exhibits non-structured patterns (smears with MNase digestion), was almost indistinguishable from that in other euchromatin regions, namely regular ladder patterns. This indicates that structural compositions of histones in this core centromere region may be altered by Ams 2 overproduction. Indeed, in these Ams2-overproducing cells, a substantial amount of $\mathrm{H} 3$ histones is incorporated into the centromere. It is important to note that in these cells CENP-A ${ }^{\text {Cnp } 1}$ still exists at the core centromere as in wild-type. That is, the number of nucleosomes is increased, in which nucleosomes comprising canonical H3 or CENP-ACnp1 


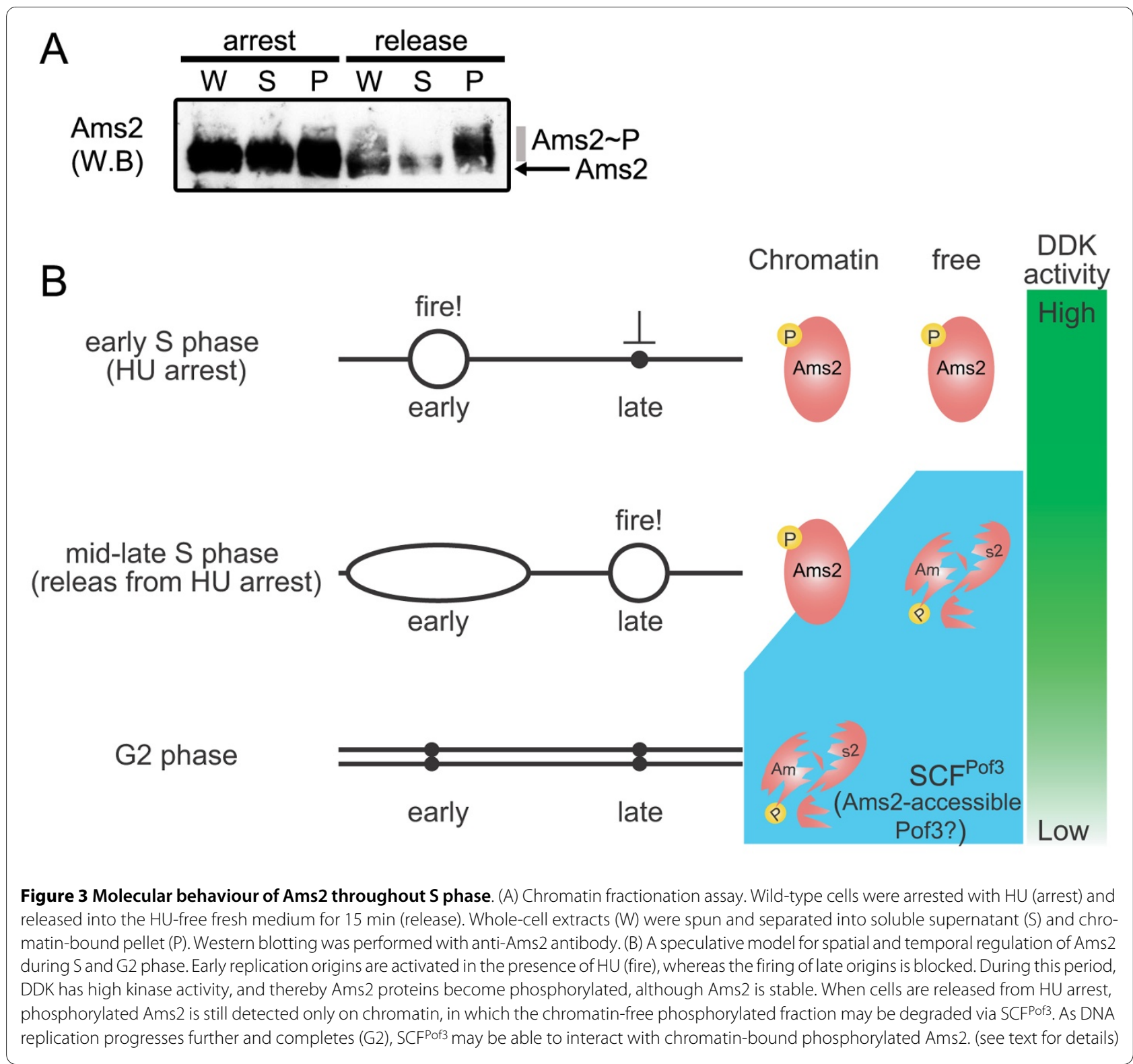

appear to coexist in the core centromere, leading to formation of more tightly packed nucleosomes in this region. Perhaps this abnormal centromere status would be the reason, at least in part, for growth arrest and massive chromosome loss phenotypes. Analysis of euchromatin (e.g., around the actin gene) in Ams2-overproducing cells also indicates that the overall number of nucleosomes is increased. In summary, induced overexpression of all the core histone genes forces formation of more nucleosomes on both euchromatin and centromere regions.

These results indicate that despite the existence of multiple mechanisms by which to ensure the formation of centromere-specific nucleosomes [10-16], overproduction of a single transcription factor, Ams2, is sufficient to interfere with these regulatory networks. We envisage that this edge of wall status of histone homeostasis is indeed the reason that fission yeast has developed DDKphosphorylation- and SCF-mediated proteolysis of Ams2 during the cell cycle. It would be interesting to address other defects except at the centromere in Ams2-overexpressing cells.

\section{Evolutionary conservation of histone homeostasis from yeast to human}

Are the mechanisms underlying cell cycle-dependent histone homeostasis conserved? The Ams2 protein, however, seems not to be conserved in other species, which may suggest that what we have found is a fission yeastspecific regulatory system. Nonetheless as described 
below, we envision that although individual organisms have developed different strategies to maintain a proper level of histones, they implement the universal regulatory system, that is, the ubiquitin-proteasome pathway.

In budding yeast, histone levels, in particular those of non-chromatin forms, are regulated by phosphorylation and the ubiquitin-proteasome pathway [36]. In this regulatory system, excess free histones are phosphorylated by Rad53 (homologues of fission yeast Cds1 and human CHK1/2), resulting in polyubiquitylation via the Ubc4/ Ubc5 (E2) and Tom1 (E3) and subsequent degradation by the proteasome (Figure 4, right). Although not shown in fission yeast, it is noteworthy that in budding yeast and fly, CENP-A protein levels are strictly regulated by the ubiquitin-proteasome system $[37,38]$. Therefore, at least in yeast, the histone levels are under the control of phosphorylation and the ubiquitin-proteasome system.
In mammalian cells, unlike yeast or plants, the 3 ' ends of histone mRNAs contain a stem-loop structure. This specific structure is recognised by stem-loop binding protein (SLBP), by which mRNA half-life is determined. Interestingly, SLBP is synthesised at S phase and is rapidly degraded at the end of S phase. Furthermore, SLBP degradation is triggered by phosphorylation through CDKcyclinA (Figure 4, left) [21,39]. However, the molecular basis of SLPB degradation is not known. Given the degradation of fission yeast Ams2 and budding yeast free histones, we envisage that SLBP is degraded via the ubiquitin-proteasome pathway. Hence, although the molecular details of regulatory systems may not be the same among individual species, the underlying principles as to how histone homeostasis is maintain via ubiquitinmediated proteolysis would be conserved from yeast to human beings.

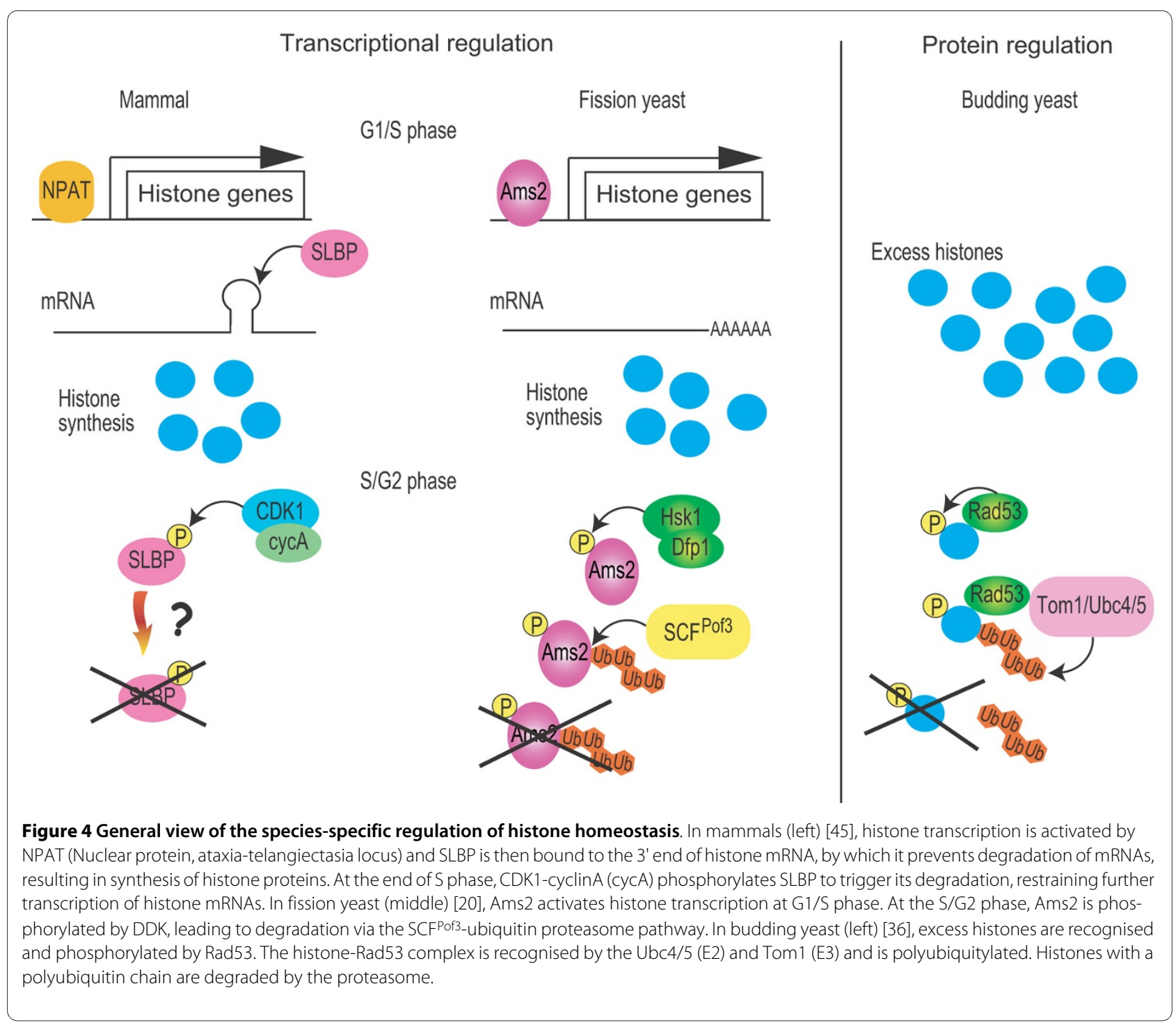




\section{Concluding remarks}

Through the pioneering work of Hartwell and colleagues [4], it is known that ectopic expression of core histone genes has a deleterious effect on chromosome transmission fidelity. However, the underlying mechanism of this toxicity has remained a mystery for decades. We have shown that in fission yeast the levels of the Ams2 transcription factor, which is responsible for periodic histone gene expression, are regulated via the $\mathrm{SCF}^{\mathrm{Pof} 3}$ ubiquitinproteasome pathway and that Ams2 proteolysis plays a critical role in maintaining chromosome stability. Importantly, constitutive synthesis of Ams2, and therefore excess core histones, results in structural alterations of centromeric nucleosomes. Our study, therefore, provides the first clear answer to the above question--this is at least in part ascribable to the impairment of centromere structure and function.

Our work illuminates interesting, unexpected parallels between CDK and DDK during the cell cycle, in particular their roles during G2 phase. It is well established that CDK is important to prevent re-replication of chromosomes upon completion of $S$ phase [40]. In contrast, it is generally believed that DDK's major, if not sole, role lies in $S$ phase, although previous work in yeast indicated that this might not always be true [41,42]. Our study clearly showed that DDK is needed to degrade Ams2 during G2 phase, thereby preventing unnecessary histone gene expression during the post-S phase period. Hence, CDK and DDK not only share roles in S phase initiation and progression, but are also required to secure proper passage of G2 phase upon completion of S phase. CDK is required for restraining deleterious re-replication of the genome, whereas DDK prevents extra histone transcription, which would result in global and local genome disturbance, including centromere malfunction (Figure 5).

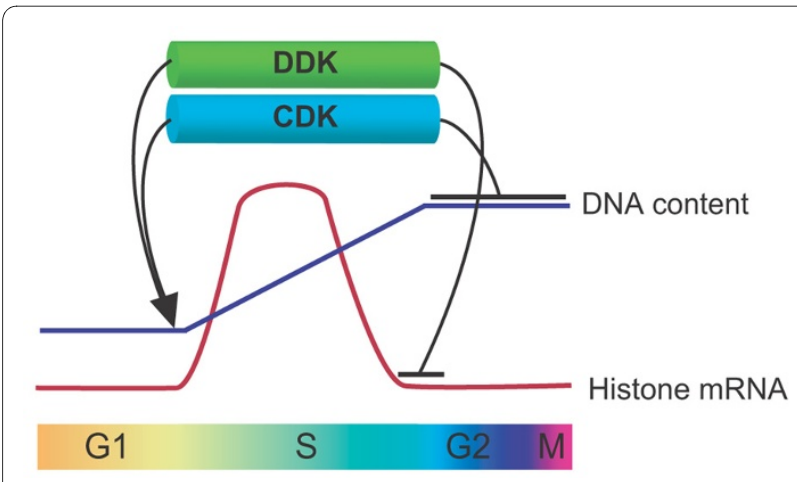

Figure 5 Cooperative roles of CDK and DDK in cell cycle progression from $\mathbf{G} 1$ to $\mathbf{G} 2$ phase. CDK and DDK promote G1-S phase transition and are required for proper $S$ phase progression (arrows). In addition, these two kinases act in concert during G2 phase. CDK is required for prevention of chromosomal DNA re-replication, whereas DDK is essential to repress untimely histone gene expression, thereby helping ensure centromere integrity.
Cooperative action between CDK and DDK is consistent with their structural and regulatory framework. Both kinases consist of catalytic and regulatory subunits, in which their regulatory subunits play a decisive role in kinase activities, localisation and substrate recognition. Intriguingly, the levels of cyclins (for CDK) and Dbf4/ Dfp1 (for DDK) are under the control of the APC/C ubiquitin ligase $[43,44]$. Although phylogenetically neither catalytic nor regulatory subunit of CDK and DDK is evolutionarily close, common regulatory mechanisms suggest that CDK and DDK are, at least functionally, much more intimate and cooperative than currently thought.

\section{List of abbreviations}

APC/C: Anaphase Promoting Complex/Cyclosome CDK: cyclin-dependent kinase; DDK: Dbf4-dependent kinase; E1: ubiquitin-activating enzyme; E2: ubiquitin-conjugating enzyme; E3: ubiquitin ligase; HU: hydroxyurea; MNase: Micrococcal nuclease; LRR: leucine rich repeats; NPAT: Nuclear protein; ataxia-telangiectasia locus; SCF: Skp1-Cdc53/Cullin-1-F-box; SLBP: stem-loop binding protein; TPR: tetratricopeptide repeat.

\section{Competing interests}

The authors declare that they have no competing interests.

\section{Authors' contributions}

YT designed and performed experiments and prepared the figures. YT and TT wrote the paper. Both authors have read and approved the final manuscript

\section{Acknowledgements}

We thank Shigeaki Saitoh for valuable help and suggestions during this work, and Hisao Masukata for discussion. We are also grateful to Hiroyuki Yamano for informing us of his unpublished data. This work was supported by Cancer

Research UK (T.T.) and Grant-in-aid for Young Scientists (B) from the Japan Society for the Promotion of Science (Y.T.)

\section{Author Details}

1Division of Cell Biology, Institute of Life Science, Kurume University, 1-1 Hyakunen-kohen, Kurume, Fukuoka 839-0864, Japan and 2Laboratory of Cell Regulation, London Research Institute, Cancer Research UK, 44 Lincoln's Inn Fields, London WC2A 3PX, UK

Received: 29 June 2010 Accepted: 7 July 2010

Published: 7 July 2010

References

1. Hershko A, Ciechanover A, Varshavsky A: Basic Medical Research Award. The ubiquitin system. Nat Med 2000, 6(10):1073-1081.

2. Ravid T, Hochstrasser M: Diversity of degradation signals in the ubiquitin-proteasome system. Nat Rev Mol Cell Biol 2008, 9(9):679-690.

3. Gunjan A, Paik J, Verreault A: The emergence of regulated histone proteolysis. Curr Opin Genet Dev 2006, 16(2):112-118.

4. Meeks-Wagner D, Hartwell LH: Normal stoichiometry of histone dimer sets is necessary for high fidelity of mitotic chromosome transmission. Cell 1986, 44(1):43-52

5. Gunjan A, Verreault A: A Rad53 kinase-dependent surveillance mechanism that regulates histone protein levels in S. cerevisiae. Cell 2003, 115(5):537-549.

6. Lengauer C, Kinzler KW, Vogelstein B: Genetic instability in colorectal cancers. Nature 1997, 386(6625):623-627.

7. Ricke RM, van Ree JH, van Deursen JM: Whole chromosome instability and cancer: a complex relationship. Trends Genet 2008, 24(9):457-466. 
8. Black BE, Bassett EA: The histone variant CENP-A and centromere specification. Curr Opin Cell Biol 2008, 20(1):91-100.

9. Buscaino A, Allshire R, Pidoux A: Building centromeres: home sweet home or a nomadic existence? Curr Opin Genet Dev 2010, 20(2):118-126.

10. Sanchez-Pulido L, Pidoux AL, Ponting CP, Allshire RC: Common ancestry of the CENP-A chaperones Scm3 and HJURP. Cell 2009, 137(7):1173-1174.

11. Dunleavy EM, Roche D, Tagami H, Lacoste N, Ray-Gallet $D$, Nakamura $Y$, Daigo Y, Nakatani Y, Almouzni-Pettinotti G: HJURP is a cell-cycledependent maintenance and deposition factor of CENP-A at centromeres. Cell 2009, 137(3):485-497.

12. Foltz DR, Jansen LE, Bailey AO, Yates JR, Bassett EA, Wood S, Black BE, Cleveland DW: Centromere-specific assembly of CENP-A nucleosomes is mediated by HJURP. Cell 2009, 137(3):472-484.

13. Dunleavy EM, Pidoux AL, Monet M, Bonilla C, Richardson W, Hamilton GL, Ekwall K, McLaughlin PJ, Allshire RC: A NASP (N1/N2)-related protein, Sim3, binds CENP-A and is required for its deposition at fission yeast centromeres. Mol Cell 2007, 28(6):1029-1044.

14. Hayashi T, Fujita Y, Iwasaki O, Adachi Y, Takahashi K, Yanagida M: Mis16 and Mis 18 are required for CENP-A loading and histone deacetylation at centromeres. Cell 2004, 118(6):715-729.

15. Fujita Y, Hayashi T, Kiyomitsu T, Toyoda Y, Kokubu A, Obuse C, Yanagida M: Priming of centromere for CENP-A recruitment by human hMis18alpha, hMis18beta, and M18BP1. Dev Cell 2007, 12(1):17-30.

16. Walfridsson J, Bjerling P, Thalen M, Yoo EJ, Park SD, Ekwall K: The CHD remodeling factor Hrp1 stimulates CENP-A loading to centromeres. Nucleic Acids Res 2005, 33(9):2868-2879.

17. Takayama Y, Takahashi K: Differential regulation of repeated histone genes during the fission yeast cell cycle. Nucleic Acids Res 2007, 35(10):3223-3237.

18. Takayama Y, Sato H, Saitoh S, Ogiyama Y, Masuda F, Takahashi K: Biphasic incorporation of centromeric histone CENP-A in fission yeast. Mol Biol Cell 2008, 19(2):682-690.

19. Chen ES, Saitoh S, Yanagida M, Takahashi K: A cell cycle-regulated GATA factor promotes centromeric localization of CENP-A in fission yeast. Mol Cell 2003, 11(1):175-187.

20. Takayama Y, Mamnun YM, Trickey M, Dhut S, Masuda F, Yamano H, Toda T, Saitoh S: Hsk1- and SCFPof3-dependent proteolysis of S. pombe Ams2 ensures histone homeostasis and centromere function. Dev Cell 2010 18(3):385-396

21. Marzluff WF: Terminating histone synthesis to preserve centromere integrity. Dev Cell 2010, 18(3):335-336

22. Takahashi K, Chen ES, Yanagida M: Requirement of Mis6 centromere connector for localizing a CENP-A-like protein in fission yeast. Science 2000, 288(5474):2215-2219.

23. Peters JM: The anaphase promoting complex/cyclosome: a machine designed to destroy. Nat Rev Mol Cell Biol 2006, 7(9):644-656.

24. Petroski MD, Deshaies RJ: Function and regulation of cullin-RING ubiquitin ligases. Nat Rev Mol Cell Biol 2005, 6(1):9-20.

25. Lehmann A, Katayama S, Harrison C, Dhut S, Kitamura K, McDonald N, Toda T: Molecular interactions of fission yeast Skp1 and its role in the DNA damage checkpoint. Genes Cells 2004, 9(5):367-382.

26. Tafforeau L, Le Blastier S, Bamps S, Dewez M, Vandenhaute J, Hermand D: Repression of ergosterol level during oxidative stress by fission yeast $F$ box protein Pof14 independently of SCF. EMBO J 2006, 25(19):4547-4556.

27. Hermand D: F-box proteins: more than baits for the SCF? Cell Div 2006, $1: 30$.

28. Katayama S, Kitamura K, Lehmann A, Nikaido O, Toda T: Fission yeast Fbox protein Pof3 is required for genome integrity and telomere function. Mol Biol Cell 2002, 13(1):211-224

29. Kominami K, Seth-Smith H, Toda T: Apc10 and Ste9/Srw1, two regulators of the APC-cyclosome, as well as the CDK inhibitor Rum1 are required for G1 cell-cycle arrest in fission yeast. EMBO J 1998, 17(18):5388-5399.

30. Kominami K, Toda T: Fission yeast WD-repeat protein pop1 regulates genome ploidy through ubiquitin-proteasome-mediated degradation of the CDK inhibitor Rum 1 and the S-phase initiator Cdc18. Genes Dev 1997, 11(12):1548-1560

31. Jallepalli PV, Tien D, Kelly TJ: sud 1+ targets cyclin-dependent kinasephosphorylated $\mathrm{Cdc} 18$ and Rum 1 proteins for degradation and stops unwanted diploidization in fission yeast. Proc Natl Acad Sci USA 1998, 95(14):8159-8164
32. Wolf DA, McKeon F, Jackson PK: F-box/WD-repeat proteins pop1p and Sud 1 p/Pop2p form complexes that bind and direct the proteolysis of cdc18p. Curr Biol 1999, 9(7):373-376

33. Yamano H, Kominami K, Harrison C, Kitamura K, Katayama S, Dhut S, Hunt T, Toda T: Requirement of the SCFPop1/Pop2 Ubiquitin Ligase for Degradation of the Fission Yeast S Phase Cyclin Cig2. J Biol Chem 2004, 279(18):18974-18980

34. Seibert V, Prohl C, Schoultz I, Rhee E, Lopez R, Abderazzaq K, Zhou C, Wolf DA: Combinatorial diversity of fission yeast SCF ubiquitin ligases by homo- and heterooligomeric assemblies of the F-box proteins Pop $1 \mathrm{p}$ and Pop2p. BMC Biochem 2002, 3(22):22.

35. Patton EE, Willems AR, Tyers M: Combinatorial control in ubiquitindependent proteolysis: don't Skp the F-box hypothesis. Trends Genet 1998, 14(6):236-243.

36. Singh RK, Kabbaj MH, Paik J, Gunjan A: Histone levels are regulated by phosphorylation and ubiquitylation-dependent proteolysis. Nat Cell Biol 2009, 11(8):925-933

37. Collins KA, Furuyama S, Biggins S: Proteolysis contributes to the exclusive centromere localization of the yeast Cse4/CENP-A histone H3 variant. Curr Biol 2004, 14(21):1968-1972.

38. Moreno-Moreno O, Torras-Llort M, Azorin F: Proteolysis restricts localization of CID, the centromere-specific histone $\mathrm{H} 3$ variant of Drosophila, to centromeres. Nucleic Acids Res 2006, 34(21):6247-6255.

39. Koseoglu MM, Graves LM, Marzluff WF: Phosphorylation of threonine 61 by cyclin $\mathrm{A} / \mathrm{Cdk} 1$ triggers degradation of stem-loop binding protein at the end of S phase. Mol Cell Biol 2008, 28(14):4469-4479.

40. Takeda DY, Dutta A: DNA replication and progression through S phase. Oncogene 2005, 24(17):2827-2843

41. Bailis JM, Bernard P, Antonelli R, Allshire RC, Forsburg SL: Hsk1-Dfp1 is required for heterochromatin-mediated cohesion at centromeres. Nat Cell Biol 2003, 5(12):1111-1116.

42. Matos J, Lipp JJ, Bogdanova A, Guillot S, Okaz E, Junqueira M, Shevchenko A, Zachariae W: Dbf4-dependent CDC7 kinase links DNA replication to the segregation of homologous chromosomes in meiosis I. Cell 2008, 135(4):662-678

43. Diffley JF: Regulation of early events in chromosome replication. Curr Biol 2004, 14(18):R778-786.

44. Ferreira MF, Santocanale C, Drury LS, Diffley JF: Dbf4p, an essential S phase-promoting factor, is targeted for degradation by the anaphasepromoting complex. Mol Cell Biol 2000, 20(1):242-248.

45. Marzluff WF, Wagner EJ, Duronio RJ: Metabolism and regulation of canonical histone mRNAs: life without a poly(A) tail. Nat Rev Genet 2008, 9(11):843-854

46. Kominami K, Ochotorena I, Toda T: Two F-box/WD-repeat proteins Pop1 and Pop2 form hetero- and homo-complexes together with cullin-1 in the fission yeast SCF (Skp1-Cullin-1-F-box) ubiquitin ligase. Genes Cells 1998, 3(11):721-735.

47. Harrison C, Katayama S, Dhut S, Chen D, Jones N, Bahler J, Toda T: SCFPof1_ ubiquitin and its target Zip1 transcription factor mediate cadmium response in fission yeast. EMBO J 2005, 24(3):599-610.

48. Mamnun YM, Katayama S, Toda T: Fission yeast Mcl1 interacts with SCFPof3 and is required for centromere formation. Biochem Biophys Res Commun 2006, 350(1):125-130.

49. Jourdain I, Spielewoy N, Thompson J, Dhut S, Yates JR, Toda T: Identification of a conserved F-box protein 6 interactor essential for endocytosis and cytokinesis in fission yeast. Biochem J 2009, 420(2):169-177.

50. Hermand D, Bamps S, Tafforeau L, Vandenhaute J, Makela TP: Skp1 and the F-box protein Pof6 are essential for cell separation in fission yeast. J Biol Chem 2003, 278(11):9671-9677.

51. Schmidt MW, McQuary PR, Wee S, Hofmann K, Wolf DA: F-box-directed CRL complex assembly and regulation by the CSN and CAND1. Mol Cell 2009, 35(5):586-597.

52. Ikebe C, Kominami K, Toda T, Nakayama K. Isolation and characterization of a novel F-box protein Pof10 in fission yeast. Biochem Biophys Res Commun 2002, 290(5):1399-1407.

53. Kim J, Kim JH, Lee SH, Kim DH, Kang HY, Bae SH, Pan ZQ, Seo YS: The novel human DNA helicase hFBH1 is an F-box protein. $J$ Biol Chem 2002, 277(27):24530-24537.

54. Lawrence $C L$, Jones N, Wilkinson CR: Stress-induced phosphorylation of S. pombe Atf1 abrogates its interaction with F box protein Fbh1. Curr Bio/ 2009, 19(22):1907-1911. 
55. Lorenz A, Osman F, Folkyte V, Sofueva S, Whitby MC: Fbh1 limits Rad51dependent recombination at blocked replication forks. Mol Cell Biol 2009, 29(17):4742-4756.

56. Morishita T, Furukawa F, Sakaguchi C, Toda T, Carr AM, Iwasaki H, Shinagawa H: Role of the Schizosaccharomyces pombe F-Box DNA helicase in processing recombination intermediates. Mol Cell Biol 2005, 25(18):8074-8083.

57. Osman F, Dixon J, Barr AR, Whitby MC: The F-Box DNA helicase Fbh1 prevents Rhp51-dependent recombination without mediator proteins. Mol Cell Biol 2005, 25(18):8084-8096.

58. Sakaguchi C, Morishita T, Shinagawa $H$, Hishida T: Essential and distinct roles of the F-box and helicase domains of Fbh1 in DNA damage repair. BMC Mol Biol 2008, 9(27):27.

59. Schulman BA, Carrano AC, Jeffrey PD, Bowen Z, Kinnucan ER, Finnin MS, Elledge SJ, Harper JW, Pagano M, Pavletich NP: Insights into SCF ubiquitin ligases from the structure of the Skp1-Skp2 complex. Nature 2000, 408(6810):381-386.

doi: 10.1186/1747-1028-5-18

Cite this article as: Takayama and Toda, Coupling histone homeostasis to centromere integrity via the ubiquitin-proteasome system Cell Division 2010, $5: 18$

Submit your next manuscript to BioMed Central and take full advantage of:

- Convenient online submission

- Thorough peer review

- No space constraints or color figure charges

- Immediate publication on acceptance

- Inclusion in PubMed, CAS, Scopus and Google Scholar

- Research which is freely available for redistribution 